\title{
Preußisch? Protestantisch? Plebejisch? Berlins Entwicklung zu einer Metropole kritischer Gegenkultur
}

Prussienne, protestante, plébéienne. La transformation de Berlin en une métropole de la contre-culture critique

Prussian, Protestant, Plebian: Berlin's Transformation into a Metropolis of Critical Counter-Culture

Jürgen Hofmann

\section{OpenEdition}

Journals

Édition électronique

URL : https://journals.openedition.org/ceg/6599

DOI : $10.4000 /$ ceg.6599

ISSN : 2605-8359

\section{Éditeur}

Presses Universitaires de Provence

Édition imprimée

Date de publication : 1 mai 2013

Pagination : 25-38

ISBN : 0751-4239

ISSN : 0751-4239

\section{Référence électronique}

Jürgen Hofmann, „Preußisch? Protestantisch? Plebejisch? Berlins Entwicklung zu einer Metropole kritischer Gegenkultur", Cahiers d'Études Germaniques [Online], 64 | 2013, Online erschienen am: 02 Januar 2020, abgerufen am 02 Juni 2021. URL: http://journals.openedition.org/ceg/6599; DOI: https://doi.org/10.4000/ceg.6599 


\title{
Preussisch? Protestantisch? Plebejisch? Berlins Entwicklung zu einer Metropole kritischer Gegenkultur
}

\author{
Jürgen HOFMANN \\ Freier Schriftsteller, Berlin
}

Seit 1961 habe ich mich mehrere Jahrzehnte in der kritischen Gegenkultur von Berlin bewegt. Ich könnte, wie der Berliner sagt, Arien davon singen, nachts und auswendig.

Nun scheint mir von empirischen Exempeln dieser kulturellen Alternativen im vorliegenden Buch hinreichend die Rede. Also möchte ich bei meinem Beitrag einen Schritt zurücktreten, um eine neue, mir selbst ungewisse Frage näher zu untersuchen. Ich möchte darüber nachdenken, was eigentlich Berlin zu dieser Eigenschaft determiniert. Welche Qualitäten könnten es sein, die diese Stadt zu einer Metropole kritischer Gegenkultur offenbar prädestinieren?

In seiner Autobiografie Le lièvre de Patagonie („Der patagonische Hase") beschreibt Claude Lanzmann, wie er 1948 zum ersten Mal nach Berlin kommt, wo er als Lehrbeauftragter an der eben erst gegründeten Freien Universität arbeiten soll. Angesichts der Ruinen der Gestapo-Zentrale versucht der spätere Autor von Shoa die Hauptstadt des Nazi-Terrors, des Holocaust, nüchtern als Chance zu begreifen. Berlin, so konstatiert der jüdischstämmige Überlebende der Resistance, biete mit der gleichzeitigen Gegenwart aller historischen Epochen - von preussischen Königen, Wilhelminismus, Weimarer Republik und NS-Zeit bis hin zur Sektorenstadt von West-Alliierten und Kommunisten - eine einzigartig anschauliche Gelegenheit, das 20. Jahrhundert wie in einem geologischen Querschnitt zu begreifen.

Genau das umgekehrte Bild entwirft einmal Sigmund Freud in seiner ebenfalls während der Nazizeit entstandenen - Schrift über Das Unbehagen in der Kultur. Um zu veranschaulichen, dass im Unbewussten alles Vergangene permanent gegenwärtig sei, vergleicht er es mit den historischen Schichten Roms. Wenn man sich die Stadt so vorstelle, als bestïnde sie aus 
Antike, Renaissance, Barock, 19. Jahrhundert und Moderne zugleich, dann könne man auch die Dimensionen der Schichtung unserer Psyche begreifen.

Mit dieser dialektischen Beziehung von Mentalität und Geschichte will ich mich Berlin nähern - eher skizzierend-erzählerisch als systematischstringent - und meine dabei gewonnenen Beobachtungen in einigen Merksätzen festhalten. Zu diesem Zweck werde ich zuerst sozusagen per Fernrohr auf ein frühes Jahrhundert Berlins blicken - das vor der Französischen Revolution. Nach einem harten Schnitt will ich dann mit einem rapidem Zoom die Stadt betrachten, wie sie unmittelbar vor und nach dem Mauerbau aussieht.

Beginnen aber will ich zunächst mit einer Art Prolog über den Berliner und sein Theater:

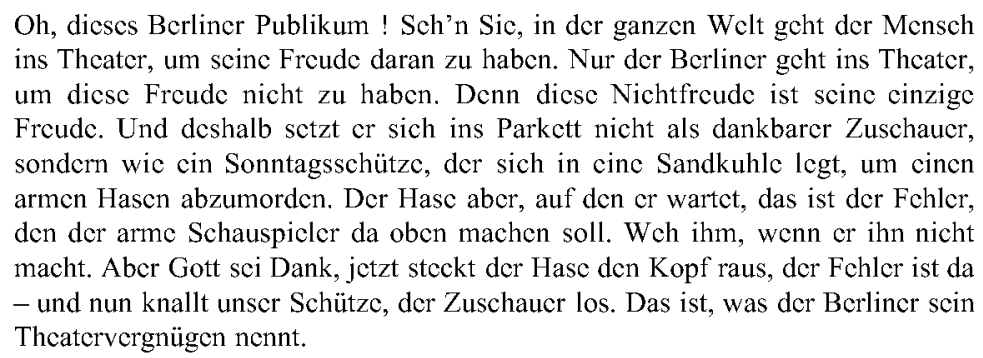

Hier beschreibt der Schauspieler Theodor Döring Mitte des 19. Jahrhunderts pointiert das ständige Kritisieren, wie es für die Berliner Mentalität äußerst typisch ist. „Da kann man nicht meckern“ gilt in Berlin als äußerster Ausdruck von Anerkennung. Der hier meckert, Theodor Fontane, ist selbst, außer als (Theater-)Kritiker, ein sehr typischer Berliner. Er kommt aus der Provinz in die Metropole - und er gehört, als bekennender Hugenotte, zu einer für die Stadt wichtigen ethnischen Minderheit.

In Deutschland wird Berlin von Auswärtigen, also den wenigen nicht nach Berlin eingewanderten Minderheiten, vor allem verknüpft mit Witz. Unter den deutschen Stämmen gilt der Berliner als schlagfertig, als nüchternkritisch - „helle“ würde man in der Metropole selbst sagen -, allerdings auch als angeberisch, großspurig. „Berlin, dein Gesicht hat Sommersprossen - und dein Mund ist viel zu groß..." - singt in einem bekannten Chanson (1966) eine berühmte Berlinerin, Hildegard Knef.

Viele Wahl-Berliner bekunden, welch starke Anziehung gerade das Berlinische mit seiner Einheit von Dialekt und Witz auf sie von Anfang an ausgeübt hat. Fritz Kortner, der nachmals berühmte Berliner Theatermann beispielsweise, kommt als jüdischstämmiger Schauspieler erstmals 1911 aus Wien nach Berlin. In seiner lesenswerten Autobiografie beschreibt er, wie er sich augenblicklich in die Stadt und ihren Dialekt verliebt. Eines seiner Beispiele dafür ist die angeberische Drohung, die er auf der Straße aufschnappt und die man natürlich in der Mundart zitieren muss: „Ick hau'Se an die Wand, det'Se uff der anderen Seite als Relief erscheinen..." 
Fontane, Kortner, Knef: der hugenottische Kritiker, der jüdischstämmige Theatermann und die Berliner Göre - sie scheinen mir das geeignete Trio am Eingangstor zu unserem Gegenstand. An ihnen nämlich ist zweierlei ablesbar: zur Eigenheit von Berlin gehört wesentlich der Fremde, das Fremde. Und: für den Berliner gehören Erkenntnis, Witz und Kritik eng zusammen. Mein erster Merksatz heißt dementsprechend auch: Berlin ist ganz Kopf, ganz Sprache, ganz Kritik.

Dieser Berliner Rationalismus gilt einerseits vielen Menschen aus anderen Kulturen, besonders den mediterranen, als gefühllos, ja sogar als unerotisch. Der Kabarettist und Schauspieler Wolfgang Neuss hat das mal auf den Nenner gebracht, der Berliner verfüge wohl über eine Art Sentimentalsperre. Andererseits wird dieses Talent zur Kritik, der Zwang zur Nüchternheit, auch als ausgesprochen anti-autoritär empfunden, ja als Ausdruck plebejischer Vernunft.

Bemerkenswert jedenfalls erscheint mir, dass die Nazis in Berlin immer die wenigsten Anhänger von ganz Deutschland fanden. Noch bei den letzten Reichstags-Wahlen, nach dem Machtantritt der Nazis im März 1933 unter starkem Terror abgehalten, erhielt ihre NSDAP in Berlin nicht einmal ein Drittel der Stimmen. Hitler, der von Linz und Wien, München und Nürnberg schwärmte, mochte seine Reichshauptstadt nie - und die Berliner erwiderten seine Abneigung von Herzen.

Begeistert notiert der Emigrant Kortner, was ihm in den USA während des Krieges aus Berlin zugetragen wird. Dort hat Goebbels das berühmte Motiv des Werbe-Posters von Uncle Sam (,Join the Army") geklaut, um daraus ein Propaganda-Plakat für seinen Führer zu machen. Nun steht Hitler also vor den Umrissen von Großdeutschland und fragt: „Deutscher Volksgenosse das tat ich für dich. Was tust du für mich?" Schreibt ein Berliner handschriftlich darunter: „Ick zittere“.

\section{Mentalität und Geschichte}

Die Mark Brandenburg ist spät kolonisiert worden, gilt als Reichsland erst von der Mitte des 12. Jahrhunderts an. In diesem Stadium des ausgehenden Mittelalters stehen die deutschen Städte im Norden, Westen und Süden mit ihren Klöstern, Domen und Schlössern langst in Blüte, haben Gewerbe und Handel, geistliches Leben und Kunst reich entfaltet. Die Mark hingegen bleibt extrem dünn besiedelt. Ansiedlungspolitik ist das Epochen übergreifende Problem der Region - eigentlich bis heute.

1 S. Arnulf Ratings Intervicw (Anm. 1) in dicsem Band. Wichtige Dokumentationen über Neuss liefern Wolfgang NEUSS, Thicrry und Hans Magnus ENZENSBERGER, Asyl im Domizil, Rcinbcck bci Hamburg, Rowohlt 1968 und Volker KÜHN (Hrsg.), „Der totale Neuss ". Wolfgang Neuss Gesammelte Werke, Berlin, Rogner \& Bernhard bci Zwcitausendcins, 1997. 
Vom 15. Jahrhundert an herrscht im Land die Dynastie der Hohenzollern, die zwar aus Schwaben stammt, sich jedoch zum Inbegriff von allem entwickelt, was wir mit Preußen verbinden. Bereits kurz nach der Reformation bekennen die Regenten sich zu Luther, einige von ihnen dann sogar zum noch strengeren Calvin. In der Einheit von Thron und Altar bleibt Preußen bis 1918, vier Jahrhunderte lang, protestantisch. Das hat viele historische Vorteile - nicht zuletzt, wie Max Weber zu zeigen suchte, für den Kapitalismus, besonders den des 19. Jahrhunderts. Der Preis allerdings, den Berlin und die Mark dafür bezahlen, ist auch nicht gering. Es fehlt der Katholizismus mit seinen Kathedralen und dem Barock, es fehlt am Luxus von Kunst und Kultur. Kurz: Berlin fehlt es an Sinnlichkeit und Lebenslust.

Beim Vater Friedrichs des Großen, dem Soldatenkönig, ballt das ganze Defizit Preußens sich exemplarisch. Sehen wir einmal davon ab, dass seine drakonische Abrichtung des Thronfolgers dem Land einen geradezu schizophrenen König beschert hat, dessen inhumanes Despotentum sich mit heller Aufklärung leider die Waage hält. Was für unser Thema hier mehr interessiert: Friedrich Wilhelm I. schließt Oper und Theater, entlässt die Orchester, ja er verkauft an Schlössern, was er nicht vermieten kann - nur um die damit gewonnenen finanziellen Mittel in einem Staatshaushalt, der zu $80 \%$ dem Militär gewidmet ist, buchstäblich zu verpulvern.

Kunst-, ja Geistfeindlichkeit und Militarismus: leider verkörpert dieser Hohenzoller auf dem Thron alles, was wir - zurecht oder zu Unrecht - mit Preußen, dadurch auch mit Berlin historisch verbinden. Mein zweiter Merksatz lautet: Berlin steht auf kargem Land, bleibt arm an Bevölkerung und ist von protestantischem Puritanismus geprägt. Was Wunder, dass der rheinische Katholik Konrad Adenauer seine Abneigung gegen diese Mentalität einmal auf den Nenner des Aperçus bringt, hinter Berlin - er meint natürlich in Berlin - beginne Sibirien !

1680, gut hundert Jahre vor der Französischen Revolution, besteht Berlin aus rund 10000 Einwohnern. Das entspricht seinerzeit gerade mal der Zahl der Bediensteten von Schloß Versailles. Auch verglichen mit Städten im Kernland des Reichs wirkt Berlin mickrig: Hamburg hat zu dieser Zeit etwa 70 000, Köln über 40000 Bürger. Am Ende der Regierungszeit Friedrichs des Großen aber, 1786, gibt es allein schon in dessen Residenz Potsdam fast 20 000 Menschen. Und Berlin übertrumpft durch seine knapp 150000 Einwohner längst alle anderen deutschen Städte mit Abstand.

Zieht man in Betracht, dass insbesondere für die von Friedrich dem Großen permanent angezettelten Kriege sein Volk enorme Verluste an Menschenleben erlitt, so dürfe man über diese Bevölkerungszunahme zunächst staunen. Aber ihr Geheimnis ist simpel. Es besteht allein in jener Ansiedlungspolitik, bei der Friedrich schlicht den Praktiken seines Urgroßvaters folgt.

Diesem, Friedrich Wilhelm, kommen dabei ausgerechnet die Franzosen zu Hilfe. Es ist die Aufkündigung des Toleranzedikts von Nantes, mit der Ludwig XIV. 1685 bekanntlich eine Massenflucht der französischen 
Protestanten auslöst. Schätzungsweise $50 \quad 000$ Hugenotten gehen nach Deutschland, fast die Hälfte von ihnen siedelt sich in Preußen an - und unter ihnen werden ca. 6000 Berliner.

Schon bei einem fiüchtigen Blick ins Berliner Telefonbuch findet man bis heute jede Menge französischer Namen, wenn auch oft, wie im Fall Fontane, eingedeutscht. Und wenn man weiß, dass das Berlinische zu einem nicht geringen Teil aus - ebenfalls oft eingebürgerten - französischen Vokabeln und Redewendungen besteht, gewinnt meine eingangs zitierte Bemerkung zur Anziehungskraft des Dialekts eine neue Pointe.

Bedeutsam ist dabei allerdings nicht nur die Quantität der Einwanderer. Wichtiger noch erscheint mir, welche Art von Neubürger sich hier niederlässt. Am Ende von Friedrichs Regierungszeit besteht die Bevölkerung Preußens zu etwa einem Drittel aus Fremden - bei denen es sich fast ausschließlich um sog. Glaubensflüchtlinge handelt: Hugenotten aus Frankreich und der Schweiz treffen auf die sog. Böhmischen Brüder, aus Salzburg vertriebene Exilanten mischen sich unter Juden, die aus Österreich geflohen sind.

Sicherlich verstreuen jene Massen von Menschen sich zunächst über das inzwischen riesige Gebiet Preußens. Aber viele von ihnen lassen sich natürlich auch in der Hauptstadt nieder. Und vor allem im 19. Jahrhundert entwickelt sich in der Metropole ein lebhafter Zuzug aus allen Teilen des Königreichs. Die bevölkerungspolitisch bedingte religiös-kulturelle Vielfalt Preussens prägt frühzeitig die Mentalität Berlins. In der Identität der Stadt changiert sehr bald das Eigene konstitutiv mit dem Fremden. Mein dritter Merksatz wird also kaum überraschen: Berlin braucht die Fremden, zieht Fremde an - und besteht wesentlich aus Fremden.

Jemand, der von außen kommt und nicht selten auch lange außen stehen bleibt, vermag die Zustände und Gepflogenheiten, die er verlassen hat, mit den neuen, ungewohnten zu vergleichen. Besonders der Fremde, vielleicht sogar nur er, ist in der Lage, die Widersprüche seiner Gesellschaft wahrzunehmen. Die Ballung vieler Fremder verschafft dieser Gesellschaft im doppelten Sinn - ein kritisches Potential.

Schon Anfang 1700, bald nach der Ansiedlung der Flüchtlinge, finden in Berlin häufig heftige Auseinandersetzungen zwischen Einheimischen und Hugenotten statt. Viele Neubürger z.B. sind qualifizierte Handwerker, begabte Händler. Und natürlich fühlen die Berliner Zunftgenossen sich durch die nicht selten überlegene Konkurrenz bedroht, auf die sie bis hin zu handgreiflichen Attacken reagieren.

Das Zusammenleben so vieler Fremder stellt mithin hohe Anforderungen an die Toleranz aller. Dafür entwickeln sich einerseits in der Gesellschaft und durch sie selbst - Regulative. Andererseits macht die Konfliktlage politische Konzepte notwendig, erfordert ideologische Orientierungen, nicht zuletzt auch Gesetze. 
Unter Friedrich II., dem Großen, entwickelt sich beides. Politische Bedeutung gewinnt vor allem seine Einführung des Preussischen Landrechts ${ }^{2}$ sowie die zügige Entwicklung der allgemeinen Schulbildung. Dazu tritt eine relativ größere Freiheit der Presse und, am bekanntesten, die Proklamation der Religionsfreiheit.

Bei der Förderung religiöser Toleranz verstärken sich, was Friedrich anlangt, offenbar politische Notwendigkeit und persönliche Neigung. Die Maxime des Königs, die sein aufklärerisches Programm lakonisch formuliert, ist zum geflügelten Wort geworden: „in meinem Staat kann jeder nach seiner Façon selig werden ${ }^{36}$. Dieser Satz, früh populär, wird bis heute in Berlin nicht nur gern als Wertorientierung und Norm zitiert, sondern bis zu einem gewissen Grad auch gelebt.

Benötigt der Politiker Friedrich Toleranz für das Zusammenleben in seinem multikulturellen Land, so ist ihr Prinzip für den Aufklärer, den Gastgeber Voltaires, im kategorischen Imperativ des Preußen Kant philosophisch begründet. Dazu tritt ein höchst persönliches Motiv: ein homosexueller, antiklerikaler Künstler und Philosoph in Deutschland, zeitlich und räumlich fern der Französischen Revolution - so jemand bleibt schließlich Fremder auch in der eigenen Gesellschaft.

Friedrich der Große ist zweifellos die schillerndste Gestalt aus dem Hause Hohenzollern - und in Deutschland, zumal in Berlin, von jeher höchst umstritten. Das Jubiläumsjahr seines 300. Geburtstages bringt 2012 wieder, durchaus nachvollziehbar, heftige bis massive Kritik am diesem ,aufgeklärten Despoten" hervor.

Insofern Preußentum, reflexhaft identifiziert mit Militarismus und Untertanengeist, im Mittelpunkt auch dieser Kritik steht, will ich mich, mit Blick auf unser Thema, nur en passant auf Friedrich einlassen. Zweifellos hat er, was die Armee betrifft, diese in der Tradition seines soldatisch fixierten Vaters geradezu zu einer Militärmaschine ausgebaut. Daß ihr Funktionieren auf brutalem Zwang, extremer Grausamkeit, ja Sadismus beruhte, scheint nur den Despoten, nicht den Aufklärer interessiert zu haben. Als Nicht-Fachmann - gar als einer, der einst vor dem Dienst in der Bundeswehr ausgerechnet nach Berlin flüchtete - vermute ich dennoch, dass das preußische Heer nicht viel stärker von der Verblödung durch Disziplin und Drill, Pflichtbewusstsein und Gehorsam geprägt wurde als die Armeen anderer Länder.

Strittig bleibt die fundamentale Frage, inwieweit diese militärischen Denk- und Verhaltensweisen - gerade auch in ihrem Einhergehen mit Erfolgen, Eroberungen - das zivile Leben der Preußen, später des ganzen

2 Vollendet wurde das Gesetzeswerk unter seinem Neffen und Nachfolger Friedrich Wilhelm II. (1744-1797): das Allgemeine Preußische Landrecht trat am 1. Juni 1794 in Kraft, als eines der modernsten Gesetzbücher auf curopäischem Boden.

3 Rand-Verfügung des Königs zum Immediat-Bericht des Geistlichen Departements, Berlin 1740 Mai 22: Katholische Schulen und Proselytenmacherei: „Dic Religionen Müsen allo Tolleriret werden und Mus der fiscal nuhr das auge darauf haben, das keine der andern abruch Tuhe, den hier mus cin jeder nach Seiner Fasson Selich werden!“ 
Reichs, geformt haben. Gibt es einen historischen Zusammenhang zwischen jenem Ausmaß an spezieller militärischer Subordination, die man Kadavergehorsam genannt hat, und einem in Heinrich Manns berühmtem Roman beschworenen generellen Untertanengeist der Deutschen, wie er die beiden katastrophalen Weltkriege mit ermöglicht hat ?

Mir scheint bemerkenswert, dass selbst Historiker, die Friedrichs Größe widersprüchlich auf seine militärischen Erfolge zurückführen, gleichwohl das Charakteristische an seiner Persönlichkeit damit weniger in Zusammenhang bringen. Vielmehr scheinen die herausragenden Eigenschaften Friedrichs des Großen vor allem einer Art gesundem Menschenverstand beim Regierungshandeln zu entspringen, der vielleicht die legendäre Popularität des Königs - markiert u.a. durch seine quasi familiäre Betitelung als „Alter Fritz" - erklären könnte.

Friedrich, der Pomp und Prestigegehabe zeitlebens ablehnte, nahm auch als Soldat keinerlei königliche Privilegien je für sich in Anspruch. Zeugnisse seiner eher unmajestätischen, ja plebejischen Einstellung zum Leben finden sich höchst anschaulich unter anderem in den sog. Randglossen, also den lakonischen Anmerkungen des Monarchen zu Akten seiner Beamten. So, als es nach Steuererhöhungen in Berlin Demonstrationen gibt, reitet der König dazu, sieht ein Plakat mit einer gegen ihn gerichteten Karikatur hoch an einem Baum angebracht - und kommentiert es: „Niedriger hängen!“

Friedrich der Große scheint weniger als militärischer Oberbefehlshaber denn als Mann mit Humor in Preußen stilbildend gewirkt zu haben. Nicht zuletzt gilt er in seinem Witz als sehr berlinisch. Auch dieses ,niedriger hängen" ist populär geworden, wird heute noch häufig in Berlin zitiert und bedeutet einfach: Leute bauscht das Problem doch nicht so auf, bleibt auf dem Boden.

Es rächt sich die Fixierung des Deutsch nur radebrechenden Königs auf das feudale Frankreich, wodurch er wichtige kapitalistische und demokratische Entwicklungen im bürgerlichen England verkennt. Friedrich hinterlässt Preussen als weitgehend rückständigen Ständestaat. Während nach der französischen Revolution deutsche Jakobiner in Köln, Mainz, Wien rumoren, hört man in Berlin keinen Mucks. Die Aufklärung von oben bleibt in den Salons. Und während es im übrigen Reich dreißig zum Teil uralte Universitäten gibt, verfügt seine größte Stadt über keine einzige. Erst der Schock von Napoleons Besetzung Berlins löst eine fieberhafte Modernisierung aus, die u.a. 1810 zur Gründung der heutigen HumboldtUniversität führt.

Der aufgeklärt-aufklärerische König, dessen Philosophie freilich im fortschreitenden Alter stark von resignativen, ja depressiven Haltungen durchzogen erscheint, hat versäumt, das heraufkommende Bürgertum zu fördern, zu achten oder wenigsten zu beachten. Bei einem künstlerisch und philosophisch hochgebildeten Mann wie ihm erscheint besonders 
unbegreiflich, dass er einen Schiller geringschätzte, Lessing vollends missachtete, Kants Kritik nicht verstand.

Die Nachfolger Friedrichs des Großen tappen mit ihren Stiefeln diesbezüglich nur phantasielos in seinen Spuren. In Berlin entwickelt sich bis zur Reichsgründung 1871 kein liberales Bürgertum von Rang - das heißt vor allem: keines, das an der politischen Macht beteiligt wäre. Als seine parlamentarische Einbindung dann höchst zögerlich und partiell in Gang kommt, läuft der imperialistische Wilhelminismus bereits unaufhaltsam auf den Ersten Weltkrieg zu. Der Soziologe Helmut Plessner hat dafür den Begriff von Deutschland als der „verspäteten Nation“ geprägt, mit dem er düster auf die kompensatorischen Machtgelüste der Berliner Kaiser anspielt. Und mein vierter Merksatz benennt diesen fatalen historischen Umstand lakonisch: Berlin fehlt ein liberales Bürgertum von Rang (übrigens bis heute).

Lassen Sie mich, um mir den Sprung von Friedrich ins 20. Jahrhundert zu erleichtern, meinen Blick auf ein spezielles Kapitel seiner Toleranz werfen. Vielleicht fällt die Halbherzigkeit seiner aufklärerischen Haltung nirgends deutlicher auf als inbezug auf die Juden. Einerseits gewährt der König vielen von ihnen Aufenthalt - andererseits werden sie, wie Gegenstände, nach Brauchbarkeit klassifiziert, nach Vermögen taxiert und nach etatistischen Interessen geschröpft. Und geradezu blind bleibt der Anhänger des bedeutendsten franzosischen Aufklärers, Voltaire, gegen den jüdischstämmigen Aufklärer im eigenen Land, Moses Mendelssohn, dem als Juden er gar persönlich die Aufnahme in die Akademie der Wissenschaften verwehrt.

Unterdessen führen Mendelssohn und Lessing - die beiden bürgerlichen Aufklärer, deren Weitblick und Weltläufigkeit der französisch verblendete königliche Philosoph aberwitzig verkennt - ihm in seiner Hauptstadt exemplarisch vor, was religiöse Toleranz für die kommenden Epochen bedeuten könnte. Denn nicht nur um Duldung fremden Glaubens geht es ihnen, sondern um dessen gleichrangige Anerkennung - und nicht nur die von Juden, sondern ebenso die von Moslems. Keineswegs zufällig spielt Lessings Nathan der Weise, in dem der Dichter dem Philosophen-Freund ein leuchtendes Denkmal errichtet, im multireligiösen „Heiligen Land“.

Vielleicht muss man auch Friedrichs II. aufklärerische Leistung ,niedriger hängen“. Wie er die große deutsche Dichtung seiner Zeit links liegen ließ, jene beiden glänzenden Geister gerade mal zur Kenntnis nahm, so werden die exemplarischen Fremden, die Juden, in seinem Staat weder verfolgt noch gefördert. Immerhin gibt es in ihm ebensowenig antisemitische Bewegungen wie jüdische Ghettos in seinen Städten. Auch nach der mosaischen Façon darf jedermann hier selig werden.

So ist in Preußen der Boden für jenes Gleichheits-Edikt bereitet, mit dem unter dem Druck von Napoleons Modernisierungsschub die sog. Emanzipation der Juden von 1812 an endlich auch rechtlich eingeleitet wird. Preußen und Juden - nein, natürlich muss es heißen: nichtjüdische und 
jüdische Preußen - gehen von nun an enge, ja innige Verbindungen ein wie nirgends sonst im Deutschen Reich.

Beim Machtantritt der Nazis lebt rund ein Drittel aller jüdischen Deutschen in Berlin. Vier Fünftel der Berliner Theaterdirektoren sind jüdischstämmig. Ebenso die meisten in der Hauptstadt wirkenden Kritiker, die in den Jahren zuvor unablässig vor Hitler\&Co warnen, Siegfried Jacobsohn etwa oder der große Karl Kraus. Und natürlich Kurt Tucholsky ausnahmsweise mal ein echter Berliner. Ihn möchte ich, sozusagen als Bindeglied zwischen meinen beiden historischen Perioden, mit einem Bekenntnis von 1929 (leicht überarbeitet) zitieren:

\begin{abstract}
Wenn das Berlin ist: Radikalismus in Militärfragen, Unbedingtheit gegen den Stahl-und-Kohlen-Patriotismus; Haß gegen Verblödung durch bestimmte klerikale Kreise, Sabotage der Vorbereitungen zum nächsten Schlachten durch Kriegsminister und Justiz, wenn das alles „Berlin“ ist - dann sind wir und unsre Freunde im ganzen Land, dann sind wir für diese Stadt, in der immerhin Bewegung ist und Kraft und pulsierendes rotes Blut. Für Berlin. ${ }^{4}$
\end{abstract}

\title{
Berlin unmittelbar vor und nach dem Mauerbau
}

Bevor ich nun die Stadt unmittelbar vor und nach dem Bau der Mauer betrachte, um ihre Disposition für die Entstehung einer kritischen Gegenkultur von dieser Phase her zu begründen oder wenigstens zu beleuchten, möchte ich kurz die westdeutsche Gesellschaft skizzieren, wie sie sich mir in den fünfziger Jahren darstellt.

Es ist ein durch Nazizeit und Krieg zutiefst zerstörtes, gestörtes Gemeinwesen. Die gigantischen Verbrechen von Krieg und Faschismus samt der eigenen Mitschuld verdrängend, versuchen die Noch-einmalDavongekommenen, sich an einem hohlen System von Normen und Werten $\mathrm{zu}$ orientieren, das zusammengewürfelt ist aus 19. JahrhundertAntikommunismus, Freiheits-Pathos und Überlebens-Devisen einer kompromittierten Bourgeoisie. Diese Gesellschaft ist christlich-konservativ, patriarchalisch-autoritär und sexuell rigide. Streng geahndet werden außereheliche Beziehungen und Homosexualität, Gotteslästerung oder marxistische Bestrebungen. Die Rollenverteilung zwischen Frau und Mann in der Familie entspricht dem Biedermeier. Es versteht sich von selbst, dass die deutsche Frau nicht raucht und keine Hosen trägt, indes Männer kurze Haare und im Theater Schlips zu tragen haben. Während in Politik, Justiz, Universität usw. allenthalben alte Nazis wieder in Führungspositionen einrücken, findet weder in den Schulen noch in der Öffentlichkeit eine wirkliche Auseinandersetzung mit dem Faschismus statt. Die KPD wird verboten, die CDU regiert das Land zwei lange Jahrzehnte.

4 Kurt TUCHOLSKY (unter dem Pscudonym Ignaz WROBEL), Die Weltbühne, 29.03.1927. 
Mein fünfter Merksatz heißt: Das gesellschaftliche und geistige Leben in der BRD der Nachkriegsjahre ist bieder, spießig, prüde, rigide, retardiert. Es ist eine einzige Lähmung.

Die gute Nachricht: in Berlin ist alles - oder doch fast alles - anders!

Berlin, ich rede hier zunächst von der Zeit bis unmittelbar vor der Mauer, ist in die vier Sektoren der Alliierten geteilt. Bis zum August 1961 kann man mit der S-Bahn oder zu Fuß durchs Brandenburger Tor bequem von der kommunistischen Welt in die kapitalistische, vom westlichen Lager des Kalten Kriegs ins östliche spazieren.

In West-Berlin - und von diesem Teil will ich fast ausschließlich sprechen - sind die Spuren des Kriegs während der ganzen fünfziger Jahre noch so deutlich $\mathrm{zu}$ sehen wie nirgends in Westdeutschland. Überall stehen Ruinen. Als ich 1961 in der Stadt zu studieren anfange, wird ihr bedeutendster Boulevard, der Kurfürstendamm, vielfach noch von Baracken gesäumt.

Das Kapital ist schlicht desinteressiert an einer von den Russen umzingelten Stadt. Ihre Einwohnerzahl stagniert während dieses Jahrzehnts bei kaum mehr als zwei Millionen - und das, obwohl man hier rund 150000 Vertriebene aus den deutschen Ostgebieten angesiedelt hat und auch von den zuletzt jährlich 200000 Flüchtlingen aus der Ostzone bzw. der DDR viele in Berlin bleiben.

Anders als weite Teile Westdeutschlands wird Berlin fast schon traditionell sozialdemokratisch regiert. Bald nach dem populären NSEmigranten Ernst Reuter wird als Regierender Bürgermeister Willy Brandt gewählt, dem nicht zuletzt sein aktiver Widerstand gegen die Nazis die Anerkennung vieler Berliner einbringt.

$\mathrm{Zu}$ den Besonderheiten der Stadt gehört auch, dass, während im Bund das KPD-Verbot des Verfassungsgerichts gilt, das Alliierten-Statut Berlins das Kandidieren der Kommunisten auch im Westteil gewährleistet. Dafür dürfen ein Treppenwitz der Geschichte in der ehemaligen Hochburg des Militaristen Wilhelm und der Wehrmacht - Berliner nicht zur neuen Bundeswehr gezogen werden. Und selbst junge Westdeutsche, die in Berlin leben, sind hier vor dem Dienst mit der Waffe sicher.

Der Berliner, da gehen der antiklerikale Friedrich und seine Preußen Hand in Hand, war nie besonders kirchlich, kaum fromm, schon gar nicht katholisch. Die christlich fundamentierten Werte Westdeutschlands und der Bonner Republik werden hier ,niedriger gehängt”. Rasch nach dem Zusammenbruch entwickelt sich in Berlin wieder jenes Rotlicht-Milieu, für das die Metropole in den zwanziger Jahren berühmt war. Und während Homosexualität vor allem in den katholisch geprägten Ländern Süddeutschlands und am Rhein aktiv verfolgt und mit Zuchthaus bestraft wird, wird in Berlin die ebenfalls bald wiedererstandene Schwulenszene zumindest toleriert.

Und Berlin hat einen einzigartigen Platzvorteil: es gibt keine sog. Polizeistunde. Im Prinzip kann jede Kneipe schließen, wann sie will bzw. 
rund um die Uhr geöffnet bleiben. Und das geschieht auch, nicht zu knapp. Westdeutsche Touristen, in deren Städten um Mitternacht, wie der Berliner sagt, die Gehsteige hochgeklappt werden, schwärmen von dieser legendären Freiheit. Auch sie trägt zur Popularität der Eigenreklame bei, mit der die eingeschlossene Stadt sich damals Mut zu machen versucht: „Berlin ist immer noch Berlin".

Das Eingeschlossen- und Bedrohtsein einerseits, unbekümmertes Leben auf der anderen Seite: Berlin ist von jeher am besten in Widersprüchen zu beschreiben. Und ob man Widerspruch mit dem Wahl-Berliner Hegel als elementare Voraussetzung für Dialektik betrachtet, oder mit dem WahlBerliner Marx als gesellschaftlichen Antagonismus: Widerspruch jedenfalls darf als fruchtbarstes, ja schöpferisches Bewegungsprinzip des Geistes angesehen werden.

Dementsprechend geht mein sechster Merksatz: Durch ihre fundamentalen, historisch bedingten Widersprüche ruft die Stadt immer wieder eine besondere Produktivität hervor.

Berlin hat z.B. eine stark antikommunistisch gestimmte Bevölkerung wählt aber, verglichen mit der Bundesrepublik, notorisch relativ links. Berlin, traditionell protestantisch orientiert, tendiert eigentlich nicht $\mathrm{zu}$ rheinischer oder süddeutscher Lebensfreude - aber gegen die Grauzonen von Prostitution, Homosexualität, Cabaret mit Nackttanz und dergleichen polemisiert hierzulande noch nicht einmal die Kirche. Selbst die gegenwärtige Werbeparole der Stadt - ,arm aber sexy“ - scheint diese Tradition für ihren effektvollen Widerspruch auszubeuten.

In die zuvor beschriebene historische Situation platzt dann jenes weltpolitische Ereignis, das das Leben in Berlin und die Rolle der Stadt im Ganzen grundlegend verändert: der Bau der Mauer, am 13. August 1961. Was er konkret für viele von uns bedeutete, will ich versuchen durch eine Passage aus einer meiner Erzählungen zu veranschaulichen, die ich zum Thema '68 in Berlin geschrieben habe.

Seit die Mauer West-Berlin tückisch umzingelte, waren die entnervten Bewohner des bedrohten Atolls zu Zehntausenden abgewandert. In die verlassenen Stadtteile, die entleerten Wohnungen strömte ein buntes Völkchen, das sich gerade angezogen fühlte von der Unbehaustheit der künstlichen Insel: wir. Wehrdienstverweigerer, Rothaarige, Homosexuelle, Ostermarschierer, Linkshänder, Gammler und Atheisten - schwarze Schafe jeder Sorte „von der Etsch bis an den Belt" trafen auf die aus der Zone Abgehauenen, überhaupt auf alle, die vor etwas flüchteten: vor dem Elendsglück von Vatermutterkind, den Jägerzäunen und Stores der Reihenhäuser, vor Ulbricht, Adenauer, heilgeheuchelter Welt aller Art. Während ganz Westdeutschland emsig beschäftigt war mit „wieder" - Wiederaufbau, Wiedergutmachung, Wiederbewaffnung, Wieder-wer-sein - war in Berlin der Krieg noch allgegenwartig und die Zentralen des Terrors lagen im Schutt täglich vor uns. Nur in der schmuddligen Ruine dieser zerrissenen Stadt sahen wir die 
ungesehminkte Visage cines versehwundenen Vaterlands, dem die richtigen Fragen in dic Fresse zu schleudern wir noch nicht imstande waren. ${ }^{5}$

Der Schock, den die Mauer bei den Berlinern auslöste, saß tief. Er mobilisierte Ängste, wie die Luftbrücke sie 1949 hinterlassen und das Chruschtschow-Ultimatum zehn Jahre später sie erneuert hatte. Beträchtliche Teile der arbeitenden Bevölkerung, vor allem jüngere Familien, hauen sofort ab. Viele Unternehmer folgen.

Mit einem Schlag fehlen der Stadt die zuletzt rund 50000 Pendler, die täglich aus dem Osten zur Arbeit in den Westen fuhren. Dazu tritt der Verlust an Arbeitskräften durch die Abwanderung. Der Senat reagiert umgehend. Schon im Herbst ' 61 beginnt man in ganz Westdeutschland um Neu-Berliner zu werben. Vor allem aber - eine Aktion mit unüberschaubaren Folgen - wird ein Abkommen mit der Türkei geschlossen, das den massenhaften Nachschub von sog. Gastarbeitern sichert.

All das kann die wirtschaftlich ramponierte Inselstadt natürlich kaum aus eigener Kraft finanzieren. Enorme Zuschüsse werden aus Bonn nach Berlin gepumpt und in Förderprogramme ohne Ende umgesetzt: Umzugsbeihilfen, Möbeldarlehen, Freiflüge, Wohngeld, Weihnachtsgeld und vieles andere mehr - das die Berliner Zitterprämien nennen und bereitwillig abkassieren. Bis in die achtziger Jahre wird der Subventions-Anteil des Bundes am SenatsBudget bis auf 55\% steigen. Berlin hängt, wie man auch achselzuckend sagt, am Tropf.

Da die Stadt kaum über wirtschaftliche Perspektiven verfügt, entwickeln die Politiker ein bereits in den Schubladen vorrätiges Konzept der Metropole weiter, wonach Berlin als kulturelles Schaufenster westlicher Freiheit hergerichtet werden soll. Dabei gilt es vor allem, vorhandene bürgerliche Institutionen von Kunst und Wissenschaft auszubauen - Musiktheater, Orchester, Filmfestspiele, Universitäten, Museen usw. - und neue kulturelle Attraktionen zu schaffen. Geld? Kein Problem: Bonn zahlt.

In rascher Folge werden architektonisch beeindruckende Gebäude wie die von Oper oder Philharmonie eingeweiht. Nicht zuletzt mit verlockenden finanziellen Angeboten holt man Prominenz in die Stadt, Karajan etwa als Chefdirigenten der Philharmoniker. Auch die neue Lesungsreihe internationaler Schriftsteller in der Kongresshalle glänzt mit berühmten Namen. Das Literarische Colloquium wird erfunden, das Theatertreffen. Schließlich gelingt es gar, einen Piscator für Berlin zurückzugewinnen, der mit politisch-provokantem Theater sofort wieder seine alte Pranke zeigt.

All das zieht natürlich jede Menge von auswärtigen Künstlern und Intellektuellen an, von den namenlosen Studenten nicht zu reden. Unter ihnen sind, wie es dem Bild Berlins entspricht, die Querköpfe, die Individualisten und Dissidenten reichlich vertreten. Und während durch die eingefleischten Berliner, die sich nicht mehr aus der Stadt bewegen wollen, die Überalterung

5 Jürgen HOFMANN, Those were the days my friends, Erzählungen über ' 68 in Berlin, Berlin, 2008, S. 167. 
- samt latentem Antikommunismus - zunimmt, verjüngt die Stadt sich zugleich stetig durch ihre Neubürger. Dass nach dem Mauerbau die CDU bei den kurz darauf stattfindenden Bundestags-Wahlen mit ihrem greisen Kanzler noch einmal an die Macht kommt, verstärkt bei vielen westdeutschen Kritikern der sog. Adenauer-Ära den Drang, dieser schier endlosen Restaurations-Phase der BRD durch Flucht nach Berlin zu entrinnen.

Zum Alltag des West-Berliners gehört natürlich auch Ost-Berlin, selbst wenn es seit der Betonierung der Grenze etwas umständlicher zu erreichen ist - überdies militante Gegner des Zonen-Regimes, die vor den Stationen der dazu geeigneten S-Bahn postiert sind, noch monatelang die Berliner von der Benützung des preiswerten Verkehrsmittels handgreiflich abzuhalten suchen, nur weil es - auch einer der vielen Alltags-Widersprüche - in ganz Berlin unter Verwaltung der DDR steht.

Vieles erwirbt der Westler in Ost-Berlin preiswerter: Essen, Schnaps, Bücher und natürlich Theaterkarten. Solange auf den westlichen Bühnen noch immer nicht die eigenen gesellschaftlichen Probleme verhandelt, obendrein Brechts Stücke zur Strafe für die Mauer boykottiert werden, ist das, was gespielt wird im Deutschen Theater oder in Brechts Berliner Ensemble, oft immerhin von aktuellem Interesse. Denn wiewohl bedroht durch die Zensur des Politbüros, versuchen engagierte jüngere Autoren der DDR nachdrücklich, die großen Konflikte, wie sie im geteilten Land auf der Straße liegen, auf die Bühne zu bringen.

Und die Politik schwappt herüber und hinüber: keine andere Stadt der Welt bringt ein solch brisantes Kuriosum zustande, wie das kleine Studentenwohnheim Siegmundshof am Rande von Charlottenburg. Zu einer Lesereihe, bald nach dem Mauerbau, vermag es namhafte Schriftsteller aus Ost-Berlin als Gäste zu präsentieren. Vor stets rammelvollem Saal treten Uwe Johnson, Volker Braun, Christa Wolf und andere in Vortrag und Diskussion die heikle Gratwanderung an, zwischen vorsichtiger Kritik an ihrem Staat und demonstrativer Solidarisierung mit ihm - um dann, noch vor Mitternacht, über drei S-Bahn-Stationen pünktlich zurückzukehren in den anderen Tell der Stadt, der wie ein anderer Erdteil erscheint.

Dieser andere Stadtteil ist anders durch seine Zugehörigkeit zum anderen Lager des Kalten Kriegs, anders im System von Politik, Ideologie und Ökonomie. Aber schon im Alltag, den man beim kurzen Besuch per Passierschein wahrnimmt, erlebt man eine andere Welt: keine Werbung, keine Waren, sichtbar dürftige Verhältnisse, dafür optimistische politische Parolen ohne Ende.

Wer in dieser zerrissenen Stadt lebt - zumal als West-Berliner, der beide Welten real durchmessen kann -, in dessen Kopf bildet sich bald eine Art montiertes Bewusstsein aus, eine widersprüchliche Mischung aus Glamour und Armut, Surrealität und Subkultur samt den Lügen beider Seiten. Die Komik schlägt um in Absurdität, die Empörung in Aktion. Und aus allem erwächst ein unendliches Potential an Phantasie. 
Dieses zu pointieren will ich zum Schluß dem Komödianten Wolfgang Neuss überlassen, einem der wichtigsten Exponenten der kritischen Gegenkultur West-Berlins. Er bringt ihre Grundlagen 1983 auf einen lakonischen Nenner.

Dic Schandmaucr, cine Bauhaus-Produktion aus dem Hause Stasi, sic steht immer noch. Aber sie hat auch ihr Gutes. Seit es sic gibt, sind wir eine einzige Großfamilic geworden in West-Berlin. Sic hat unser Bewusstsein geöffnet, stachelt unser Lebensgefühl an. Was nicht mal der Zweite Weltkrieg geschafft hat, die Mauer hat's gebracht: dic ckelhaftesten Leute haben dic Stadt verlassen - nur noch dic verrücktesten bleiben in Berlin. ${ }^{6}$

Und an dieser Stelle müsste jetzt eine Drehorgel einsetzen, um eines der beliebtesten zeitlosen Berlin-Lieder zu intonieren:

Du bist verrückt mein Kind, du musst nach Berlin

Da wo dic Verrückten sind - da gehörst du hin.

6 Volker KÜHN (Hrsg.), ,Der totale Neuss “, a.a.O., S.

7 Bekannter Berliner Gassenhauer. Dic Melodic ist ein Marsch aus der selten gespielten Operette Fatinitza (1876) von Franz von Suppé. 